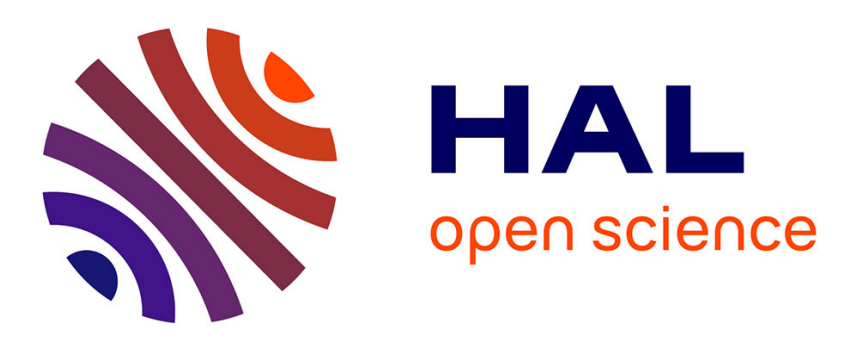

\title{
Simultaneous reconstruction of absorption, scattering and anisotropy factor distributions in quantitative photoacoustic tomography
}

Fatmir Asllanaj, Ahmad Addoum

\section{- To cite this version:}

Fatmir Asllanaj, Ahmad Addoum. Simultaneous reconstruction of absorption, scattering and anisotropy factor distributions in quantitative photoacoustic tomography. Biomed.Phys.Eng.Express, 2020, 6 (4), pp.045010. 10.1088/2057-1976/ab90a0 . hal-02870842

HAL Id: hal-02870842

https://hal.science/hal-02870842

Submitted on 22 Nov 2020

HAL is a multi-disciplinary open access archive for the deposit and dissemination of scientific research documents, whether they are published or not. The documents may come from teaching and research institutions in France or abroad, or from public or private research centers.
L'archive ouverte pluridisciplinaire HAL, est destinée au dépôt et à la diffusion de documents scientifiques de niveau recherche, publiés ou non, émanant des établissements d'enseignement et de recherche français ou étrangers, des laboratoires publics ou privés. 


\title{
Simultaneous reconstruction of absorption, scattering and
}

\section{anisotropy factor distributions in quantitative photoacoustic}

\section{tomography}

\author{
Fatmir Asllanaj ${ }^{1,2 *}$, Ahmad Addoum ${ }^{3}$ \\ ${ }^{1}$ Université de Lorraine, LEMTA, UMR 7563, Vandœuvre-lès-Nancy, F-54500, France \\ ${ }^{2}$ CNRS, LEMTA, UMR 7563, Vandœuvre-lès-Nancy, F-54500, France \\ ${ }^{3}$ Université de Lyon, IP2I, UMR 5822, Lyon, France \\ * Correspondence author - Phone : +33 372744273 \\ E-mail: Fatmir.Asllanaj@univ-lorraine.fr
}

\begin{abstract}
We present for the first time the simultaneous reconstruction of three optical parameters distributions of biological tissues namely, the absorption $\mu_{a}$ and scattering $\mu_{s}$ coefficients, as well as the anisotropy factor $g$ of the Henyey-Greenstein phase function as a new optical contrast. The 2D images are obtained from the simulation experiments and multi-source quantitative photoacoustic tomography with the radiative transfer equation (RTE) as light transport model. The image reconstruction method is based on a gradient-based optimization scheme. The adjoint method applied to the RTE is used to efficiently compute the gradient of the objective function. The results show simultaneous reconstructions of the three optical properties even with noisy data. The crosstalk problem between the three parameters is highlighted. Superior quality images are obtained for $\mu_{a}$ compared to those of $\mu_{s}$ and $g$. Moreover, our algorithm allows reconstructing inserts-like heterogeneities with very good spatial resolution and qualitative accuracy.
\end{abstract}

Keywords quantitative photoacoustic tomography, radiative transfer equation, inverse problem, image reconstruction, adjoint method, biological tissues. 


\section{${ }_{24}$ Nomenclature}

${ }_{25} A$

$26 \quad M$

${ }_{27} N_{S}$

$28 \quad g$

$29 \quad H$

$30 \quad J$

${ }_{31} n$

$32 p$

33

$34 \quad S$

35

36

Greek symbols

37

38 $\Delta \Omega$

39 $\mathcal{L}$

${ }_{40} \quad \mu_{a}$

${ }_{41} \Omega$

${ }_{42} \mu_{t}$

$43 \psi$

${ }_{44} \mu_{s}$

$45 \rho$

${ }_{46} \mathcal{R}$

$47 \Theta$

$48 \theta$

${ }_{49} \Upsilon$ absorbed energy density field, $\mathrm{W} \mathrm{mm}^{-3}$

measured data of the absorbed optical energy density, $\mathrm{W} \mathrm{mm} \mathrm{m}^{-3}$

number of collimated sources

anisotropy factor of the Henyey-Greenstein phase function

observation equation

objective function

outward unit vector normal to the medium boundary

scattering phase function

spatial position $(=x, y), \mathrm{mm}$

source term in the RTE

radiance, $\mathrm{W} \mathrm{mm}{ }^{-2} \mathrm{sr}^{-1}$

control solid angle

Lagrangian

absorption coefficient, $\mathrm{mm}^{-1}$

direction vector

attenuation coefficient $\left(=\mu_{a}+\mu_{s}\right), \mathrm{mm}^{-1}$

radiance, $\mathrm{W} \mathrm{mm}{ }^{-2} \mathrm{sr}^{-1}$

scattering coefficient, $\mathrm{mm}^{-1}$

directional reflection coefficient

state equation

angle between two directions, rad

parameter to be reconstructed

intensity of the collimated laser beam, $\mathrm{W} \mathrm{mm}-2$ 


\begin{tabular}{|c|c|c|}
\hline 50 & $\Phi$ & fluence rate, $\mathrm{W} \mathrm{mm}^{-2}$ \\
\hline 51 & $\phi$ & adjoint variable \\
\hline 52 & $\Sigma$ & interval $[0,2 \pi]$ \\
\hline 53 & $\mathcal{D}$ & domain of $\mathbb{R}^{2}$ \\
\hline 54 & $\partial \mathcal{D}$ & boundary of the medium $\mathcal{D}$ \\
\hline 55 & & \\
\hline 56 & Subscripts & \\
\hline 57 & $\mathcal{D}$ & spatial \\
\hline 58 & $\Omega$ & angular \\
\hline 59 & $\mathcal{D} \Omega$ & spatial-angular \\
\hline 60 & $c$ & collimated \\
\hline 61 & $s$ & scattered \\
\hline 62 & $s p$ & specular \\
\hline 63 & $x, y, z$ & $(O x)$-axis, $(O y)$-axis, $(O z)$-axis \\
\hline 64 & Superscripts & \\
\hline 65 & $*$ & adjoint operator \\
\hline 66 & + & incoming boundary \\
\hline 67 & - & outgoing boundary \\
\hline
\end{tabular}

\section{1 Introduction}

Photoacoustic tomography (PAT) is an emerging technique for non-invasive imaging of biological tissues. It is based on the photoacoustic effect, which refers to the generation of acoustic waves by the absorption of the optical energy in the tissue illuminated by an ultrashort pulsed laser [1-13]. The hybrid modality of PAT combines the high tissue contrast of optical imaging methods and the good spatial resolution of ultrasound imaging methods. 
PAT allows imaging at depths and resolutions unprecedented for optical methods. The optical part of PAT provides information on the distribution of chromophores, which are light-absorbing molecules within the tissue. The chromophores of interest are, for example, haemoglobin, melanin, collagen, and various contrast agents. PAT has been successfully applied to the visualization of different structures in biological tissues, such as microvasculature of tumors, human blood vessels, the cerebral cortex in small animals or breast cancer. However, this information is only a qualitative image and it does not give quantitative information on the concentrations of the chromophores. Quantitative photoacoustic tomography (QPAT) is a technique aimed at estimating the absolute concentration of the chromophores from a reconstructed PAT image. It reconstructs the optical parameters of biological tissue from data describing the absorbed energy distribution inside the tissue (assumed to be known in this work). The development of improved image reconstruction algorithm in QPAT constitutes a challenging problem [2]. An accurate forward model is essential to meet the requirements of clinical applications and to obtain a good quality reconstruction. There have been extensive studies on the optical inverse problem of QPAT, although most were using the Diffusion Equation (DE) in the diffusive regime typically assuming that the light propagation throughout the tissue is nearisotropic [7]. However, this model has well-known limitations [14-17]. In addition, the anisotropy factor also strongly affects the light propagation in tissue in the transport regime. Indeed, the biological tissues are highly forward scattering media where $g$ is typically between 0.8 and $1[9,14,18]$.

Although the absorption map is usually of the major clinic interest, it is necessary to reconstruct the scattering maps $\left(\mu_{s}, g\right)$ as well in order to accurately reconstruct the absorption map when the scattering coefficient and the anisotropy factor are unknown. Some works have shown that $g$ can be modified when the tissue is affected by a tumor because cells and cell nuclei change their size and shape. Therefore, the morphological modification of the tissue changes the scattering coefficient $\mu_{s}$. Since the anisotropy factor describes the anisotropy (angular distribution) of light scattering, this modification will also lead to a variation of $g$ values between healthy and tumor tissues. For instance, quantitative phase imaging showed, on a prostate tissue biopsy with malignancy, that the anisotropy factor $g$ can be a marker of disease [19]. Van Hillegersberg et al. [20] pointed that the anisotropy factor of rat liver decreases from 0.952 to 0.946 in a tumor at $633 \mathrm{~nm}$. Germer et al. [21] reported experimentally that $g$ was different for normal human liver tissue $(g=0.902)$ and liver metastases $(g=0.955)$ at three different 
wavelengths. Consequently, the anisotropy factor can provide an additional intrinsic contrast for optical imaging. To overcome the limitations of the DE, the Radiative Transfer Equation (RTE) has been addressed as a rigorous model for light transport in biological tissues and has become a focus of investigations in QPAT [15-17,22-35]. In the optical inverse problem of QPAT, it has been shown that the absorption coefficient $\mu_{a}$ can be reconstruct if one light source is used whereas the simultaneous reconstruction of $\left(\mu_{a}, \mu_{s}\right)$ needs multiple optical illuminations, so-called multi-source QPAT [16, 25, 36, 37]. Multiple measurements are often needed as well to eliminate nonuniqueness of the reconstruction problem [7,23].

To the best of our knowledge, the simultaneous reconstruction of $\left(\mu_{a}, \mu_{s}, g\right)$ has not been presented so far while the anisotropy factor is an important optical parameter [38]. In practical applications, the anisotropy factor is usually not known while this factor should be known to better describe light propagation. Recovering $\left(\mu_{s}, g\right)$ is especially difficult due to the weak dependence of the absorbed optical energy density on scattering. To overcome this problem, the approach has been to assume the anisotropy factor as known and estimate simultaneously $\left(\mu_{a}, \mu_{s}\right)$ in QPAT based on the RTE $[16,17,26,29,31-33]$. However, this approach can bias the estimated value of $\mu_{s}$ and also of $\mu_{a}$. In this regard, we investigated the simultaneous reconstruction of $\left(\mu_{a}, \mu_{s}, g\right)$ in multi-source QPAT based on the RTE in the transport regime. For the inversion, a gradient-based scheme using the Lm-BFGS was considered to update the spatial distribution of optical parameters. In such scheme, the major challenge is the computation of the objective function gradient which is the most expensive step. Evaluating the gradient through perturbation methods is daunting and prohibitively expensive with the RTE, especially in this case where the parameters are spatially dependent. To overcome this difficulty, the adjoint method applied to the RTE [17, 26-28, 31, 35, 38-40] was used to efficiently compute the objective function gradient with respect to the three optical parameters $\left(\mu_{a}, \mu_{s}\right.$, g) regardless the number of unknowns. In this work, a two-dimensional geometry was considered.

The inverse problem in QPAT is challenging since it is ill-posed due to the unsatisfying of both conditions uniqueness and stability. The uniqueness is caused by the strong under-determination nature of the problem where the spatially unknown number to retrieve is significantly higher than the spatially absorbed energy density data. This implies that different spatial distributions of parameters can lead to identical absorbed energy density data. Moreover, the measured noise due to the experimental setup causes an instability of the solution where small noise level 
The light source $\Upsilon(\boldsymbol{r})$, given at any location point $r \in \partial \mathcal{D}$, penetrates from the outgoing into the medium. Part of it propagates through the medium without being deviated, while the rest is scattered in all directions. It is thus convenient to split the radiance $\psi$ into two components [41]. These are denoted $\psi_{c}(\boldsymbol{r})=\psi(\boldsymbol{r}, \boldsymbol{\Omega}) \delta\left(\boldsymbol{\Omega}-\boldsymbol{\Omega}_{\boldsymbol{c}}\right)$ for $\boldsymbol{r} \in \mathcal{D}$ ( $\delta$ is the Dirac-delta function and $\boldsymbol{\Omega}_{\boldsymbol{c}}$ is the direction of the collimated laser beam) and $\psi_{s}(\boldsymbol{r}, \boldsymbol{\Omega})$ for 
$(\boldsymbol{r}, \boldsymbol{\Omega}) \in \mathcal{D} \times \Sigma$. They are respectively the collimated and scattered components of radiance [42]. The $\psi_{c}(\boldsymbol{r})$ collimated radiance is governed by the Bouguer-Beer-Lambert equation with its boundary conditions [42]:

$$
\begin{gathered}
{\left[\boldsymbol{\Omega}_{c} \cdot \nabla+\mu_{t}(\boldsymbol{r})\right] \psi_{c}(\boldsymbol{r})=0 \text { for } \boldsymbol{r} \in \mathcal{D},} \\
\psi_{c}(\boldsymbol{r})-\Upsilon(\boldsymbol{r})=0 \text { for } \boldsymbol{r} \in \partial \mathcal{D}_{c} \text { and } \psi_{c}(\boldsymbol{r})=0 \text { for } \boldsymbol{r} \in \partial \mathcal{D} \backslash \partial \mathcal{D}_{c} .
\end{gathered}
$$

where $\mu_{t}$ is the sum of the absorption and scattering coefficients. The scattered radiance $\psi_{s}(\boldsymbol{r}, \boldsymbol{\Omega})$ at location $r \in \mathcal{D} \subset \mathbb{R}^{2}$ in direction $\Omega \in \Sigma$ is solution of the steady state RTE:

$$
\left[\boldsymbol{\Omega} \cdot \nabla+\mu_{t}(\boldsymbol{r})\right] \psi_{s}(\boldsymbol{r}, \boldsymbol{\Omega})-\mu_{s}(\boldsymbol{r}) \int_{\Omega^{\prime}=2 \pi} p\left(\boldsymbol{\Omega}^{\prime} \cdot \boldsymbol{\Omega}\right) \psi_{s}\left(\boldsymbol{r}, \boldsymbol{\Omega}^{\prime}\right) d \Omega^{\prime}-S_{c}(\boldsymbol{r}, \boldsymbol{\Omega})=0
$$

for $(\boldsymbol{r}, \Omega) \in \mathcal{D} \times \Sigma$ where $S_{c}$ is an additional radiation source term to the RTE due to the scattered part of the collimated laser beam within the medium [42]:

$$
S_{c}(\boldsymbol{r}, \boldsymbol{\Omega})=\mu_{s}(\boldsymbol{r}) p\left(\boldsymbol{\Omega}_{\boldsymbol{c}} \cdot \boldsymbol{\Omega}\right) \psi_{c}(\boldsymbol{r})
$$

The Henyey-Greenstein (H-G) phase function is the most widely-adopted scattering phase function of biomedical optics [14] and this has been used here. This function depends only on the inner product between the incident direction $\Omega^{\prime}$ that scattered $\Omega$ and the anisotropy factor $g$. It is expressed for 2D media as:

$$
p\left(\boldsymbol{\Omega}^{\prime} \cdot \boldsymbol{\Omega}\right)=\frac{1}{2 \pi} \frac{1-g^{2}}{\left(1+g^{2}-2 g \boldsymbol{\Omega}^{\prime} \cdot \boldsymbol{\Omega}\right)} .
$$

The tissue surfaces are assumed to be semi-transparent boundaries due to the refractive index mismatch between air and tissue. Thus, the boundary conditions for the scattered radiance are [43]:

$$
\psi_{s}(\boldsymbol{r}, \boldsymbol{\Omega})-\frac{1}{\pi} \int_{\boldsymbol{\Omega}^{\prime} \cdot \boldsymbol{n}>0} \rho\left(\Theta^{\prime}\right) \psi_{s}\left(\boldsymbol{r}, \boldsymbol{\Omega}^{\prime}\right) \boldsymbol{\Omega}^{\prime} \cdot \boldsymbol{n} d \Omega^{\prime}=0 \text { with } \cos \Theta^{\prime}=\boldsymbol{\Omega}^{\prime} \cdot \boldsymbol{n} \text { (scattered reflection), }
$$


for $(\boldsymbol{r}, \Omega) \in \Gamma^{-}$. The directional reflection coefficient $\rho$ is given by Snell-Descartes laws assuming that the refractive index of the outside medium (air) is unity and that of tissue is equal to $1.4[42,43]$. The specular reflection $\boldsymbol{\Omega}_{\boldsymbol{s} \boldsymbol{p}}=\boldsymbol{\Omega}-2(\boldsymbol{\Omega} \cdot \boldsymbol{n}) \boldsymbol{n}$ is defined as the direction from which a laser beam must hit the surface. Then, after a specular reflection it travels in the direction of $\Omega$.

Similarly as for the radiance, the fluence may be separated into its collimated $\left(\Phi_{c}\right)$ and scattered $\left(\Phi_{s}\right)$ components:

$$
\Phi(\boldsymbol{r})=\Phi_{s}(\boldsymbol{r})+\Phi_{c}(\boldsymbol{r}) \text { for } \boldsymbol{r} \in \mathcal{D} \text { with } \Phi_{c}(\boldsymbol{r})=\psi_{c}(\boldsymbol{r}) \text { and } \Phi_{s}(\boldsymbol{r})=\int_{\Omega=2 \pi} \psi_{s}(\boldsymbol{r}, \boldsymbol{\Omega}) d \Omega
$$

The absorption of light in the tissue results in the absorbed energy density field:

$$
A(\boldsymbol{r})=\mu_{a}(\boldsymbol{r}) \Phi(\boldsymbol{r})=\mu_{a}(\boldsymbol{r}) \psi_{c}(\boldsymbol{r})+\mu_{a}(\boldsymbol{r}) \Phi_{s}(\boldsymbol{r}) .
$$

The function $\Phi(\boldsymbol{r})$ depends on the distribution of absorption and scattering within $\mathcal{D}$, as well as the light source. The optical forward problem in QPAT is to compute (10) when the optical properties of the biological tissue and the input light source are given.

\section{Optical inverse problem of QPAT}

\subsection{The objective function and observation equation}

The optical inverse problem of QPAT is to estimate the optical parameters of the tissue when the absorbed energy density $H$ is given. In this work, we intented to reconstruct the absorption $\mu_{a}$ and scattering $\mu_{s}$ coefficients as well as the anisotropy factor $g$. The spatial distribution of the vector of parameters $\theta=\left(\mu_{a}, \mu_{s}, g\right)$ is reconstructed by applying a nonlinear optimization technique to an objective function $J$ that is an explicit function of $\theta$. The real-value objective function describes the discrepancy between the measured absorbed energy density, $M(\boldsymbol{r})$ and the predicted numerical data, $A(\boldsymbol{r})$ (given from Eq. (10)). The objective function to be minimized, writes

$$
J(\theta)=\frac{1}{2} \sum_{s=1}^{N_{s}}\left\|\frac{A_{s}(\theta)-M_{s}}{M_{s}}\right\|_{\mathcal{D}}^{2},
$$


where $A_{s}(\theta)$ and $M_{s}$ are the predictions and measurements obtained with the $s^{\text {th }}$ collimated source, respectively while $N_{s}$ is the number of collimated sources. In (11), the fraction uses point-wise division and the norm is associated to $L^{2}(\mathcal{D})$, the space of real valued square-integrable functions on $\mathcal{D}$. In order to avoid round-off error due to the low level of the readings of the forward model, the function $J$ is normalized with respect to $M_{s}$. Note that $M_{s}$ can be small far away of the illuminated wall of the medium. But, this didn't affect the stability of the algorithm for the simulations presented further.

It can be noticed that all the mathematical development presented further can be made for one fixed collimated source. Then, we can omit the index $s$ for simplicity. The observation equation $A(\theta)$ is defined as:

$$
\begin{gathered}
A(\theta)(\boldsymbol{r})=\left(H \psi_{c, \theta}\right)(\boldsymbol{r})+\left(\widetilde{H} \psi_{s, \theta}\right)(\boldsymbol{r}) \text { for } \boldsymbol{r} \in \mathcal{D} \\
\text { with }\left(H \psi_{c, \theta}\right)(\boldsymbol{r})=\mu_{a}(\boldsymbol{r}) \psi_{c}(\boldsymbol{r}) \text { and }\left(\widetilde{H} \psi_{s, \theta}\right)(\boldsymbol{r})=\mu_{a}(\boldsymbol{r}) \int_{\Omega=2 \pi} \psi_{s}(\boldsymbol{r}, \boldsymbol{\Omega}) d \Omega
\end{gathered}
$$

To define compactly the state equation, we denote by $\mathcal{R}_{c}\left(\cdot, \psi_{c}\right)$ and $\mathcal{R}_{s}\left(\cdot, \psi_{c}, \psi_{s}\right)$ the right-hand sides in equations (3) and (5). Then:

$$
\mathcal{R}\left(\theta, \psi_{c}, \psi_{s}\right)=\left\{\mathcal{R}_{c}\left(\theta, \psi_{c}\right), \mathcal{R}_{s}\left(\theta, \psi_{c}, \psi_{s}\right)\right\}
$$

The reconstruction algorithm consists of minimizing $J$ when (14) is satisfied. The nonlinear optimization algorithm chosen in this work requires knowledge of the objective function gradient with respect to unknown parameters. To compute this gradient, the adjoint method (starting from the Lagrangian method) is introduced.

\subsection{The Lagrangian and adjoint method}

For one fixed collimated source, the Lagrangian is written in the $L^{2}$ space as $[44,45]$ :

$$
\mathcal{L}\left(\theta, \psi_{c}, \psi_{s}, \phi_{c}, \phi_{s}\right)=\frac{1}{2}\left\|\frac{\left(H \psi_{c}\right)+\left(\widetilde{H} \psi_{s}\right)-M}{M}\right\|_{\mathcal{D}}^{2}+\left\langle\phi_{c} \mid \mathcal{R}_{c}\right\rangle_{\mathcal{D}}+\left\langle\phi_{s} \mid \mathcal{R}_{s}\right\rangle_{\mathcal{D} \Omega}
$$

where the Lagrangian multipliers are: $\phi_{c}=\phi_{c}(\boldsymbol{r})$ (with $\boldsymbol{r} \in \mathcal{D}$ ) and $\phi_{s}=\phi_{s}(\boldsymbol{r}, \boldsymbol{\Omega})$ (with $(\boldsymbol{r}, \boldsymbol{\Omega}) \in \mathcal{D} \times \Sigma$ ). They are real functions that represent the adjoint variables associated to $\left(\psi_{c}, \psi_{s}\right)$. The two last terms in (15) are the inner 
products associated to $L^{2}(\mathcal{D})$ and $L^{2}(\mathcal{D} \Omega)$. It can be noticed that (which is trivial in appearance) if $\left(\psi_{c}, \psi_{s}\right)$ is the solution of the state equation (14) for the true $\theta$ parameter, then we have the identity:

$$
\mathcal{L}\left(\theta, \psi_{c}(\theta), \psi_{s}(\theta), \phi_{c}, \phi_{s}\right)=J(\theta), \text { for all } \phi_{c}, \phi_{s}
$$

By deriving this equation it yields:

$$
J^{\prime}(\theta) \delta \theta=\frac{\partial \mathcal{L}\left(\theta, \psi_{c}, \psi_{s}, \phi_{c}, \phi_{s}\right)}{\partial \theta} \delta \theta+\frac{\partial \mathcal{L}\left(\theta, \psi_{c}, \psi_{s}, \phi_{c}, \phi_{s}\right)}{\partial \psi_{c}} \frac{\partial \psi_{c}(\theta)}{\partial \theta} \delta \theta+\frac{\partial \mathcal{L}\left(\theta, \psi_{c}, \psi_{s}, \phi_{c}, \phi_{s}\right)}{\partial \psi_{s}} \frac{\partial \psi_{s}(\theta)}{\partial \theta} \delta \theta
$$

We denote the following independant quantities by:

$$
\delta \psi_{c}=\frac{\partial \psi_{c}(\theta)}{\partial \theta} \delta \theta \text { and } \delta \psi_{s}=\frac{\partial \psi_{s}(\theta)}{\partial \theta} \delta \theta
$$

Then, the adjoint variables are solutions to the following equation $[44,45]$ :

$$
\frac{\partial \mathcal{L}\left(\theta, \psi_{c}, \psi_{s}, \phi_{c}, \phi_{s}\right)}{\partial \psi_{c}} \delta \psi_{c}+\frac{\partial \mathcal{L}\left(\theta, \psi_{c}, \psi_{s}, \phi_{c}, \phi_{s}\right)}{\partial \psi_{s}} \delta \psi_{s}=0
$$

and Eq. (17) is reduced to:

$$
J^{\prime}(\theta) \delta \theta=\langle\nabla J(\theta) \mid \delta \theta\rangle_{\mathcal{D}}=\frac{\partial \mathcal{L}\left(\theta, \psi_{c}, \psi_{s}, \phi_{c}, \phi_{s}\right)}{\partial \theta} \delta \theta
$$

Using (15) and (19) we deduce that:

$$
\begin{gathered}
\left\langle\phi_{c} \mid \frac{\partial \mathcal{R}_{c}}{\partial \psi_{c}} \delta \psi_{c}\right\rangle_{\mathcal{D}}+\left\langle\phi_{s} \mid \frac{\partial \mathcal{R}_{s}}{\partial \psi_{s}} \delta \psi_{s}\right\rangle_{\mathcal{D} \Omega}+\left\langle\phi_{s} \mid \frac{\partial \mathcal{R}_{s}}{\partial \psi_{c}} \delta \psi_{c}\right\rangle_{\mathcal{D} \Omega} \\
+\left\langle\frac{\left(H \psi_{c}\right)+\left(\widetilde{H} \psi_{s}\right)-M}{M} \mid \frac{\left(H \delta \psi_{c}\right)}{M}\right\rangle_{\mathcal{D}}+\left\langle\frac{\left(H \psi_{c}\right)+\left(\widetilde{H} \psi_{s}\right)-M}{M} \mid \frac{\left(\widetilde{H} \delta \psi_{s}\right)}{M}\right\rangle_{\mathcal{D}}=0 .
\end{gathered}
$$

Using (12), we change $\left(H \psi_{c}\right)+\left(\widetilde{H} \psi_{s}\right)$ by $A$ in the second line of (21). As Eq. (21) has to be satisfied for all sensitivity directions $\delta \psi_{c}$ and $\delta \psi_{s}$, then it leads to the following set of equations (for each sensitivity directions): 


$$
\begin{gathered}
\left\langle\frac{A-M}{M} \mid \frac{\left(\widetilde{H} \delta \psi_{s}\right)}{M}\right\rangle_{\mathcal{D}}+\left\langle\phi_{s} \mid \frac{\partial \mathcal{R}_{s}}{\partial \psi_{s}} \delta \psi_{s}\right\rangle_{\mathcal{D} \Omega}=0 \\
\left\langle\frac{A-M}{M} \mid \frac{\left(H \delta \psi_{c}\right)}{M}\right\rangle_{\mathcal{D}}+\left\langle\phi_{c} \mid \frac{\partial \mathcal{R}_{c}}{\partial \psi_{c}} \delta \psi_{c}\right\rangle_{\mathcal{D}}+\left\langle\phi_{s} \mid \frac{\partial \mathcal{R}_{s}}{\partial \psi_{c}} \delta \psi_{c}\right\rangle_{\mathcal{D} \Omega}=0 .
\end{gathered}
$$

We denote $\mathcal{A}^{*}$ the adjoint operator of $\mathcal{A}$. Using its definition and Appendix A, the equations of (22) lead to:

$$
\begin{gathered}
\left\langle\left(\frac{\partial \mathcal{R}_{s}}{\partial \psi_{s}}\right)^{*} \phi_{s} \mid \delta \psi_{s}\right\rangle_{\mathcal{D} \Omega}+\left\langle\frac{H(A-M)}{M^{2}} \mid \delta \psi_{s}\right\rangle_{\mathcal{D}}=0 \\
\left\langle\left(\frac{\partial \mathcal{R}_{c}}{\partial \psi_{c}}\right)^{*} \phi_{c} \mid \delta \psi_{c}\right\rangle_{\mathcal{D}}+\left\langle\left(\frac{\partial \mathcal{R}_{s}}{\partial \psi_{c}}\right)^{\bar{*}} \phi_{s} \mid \delta \psi_{c}\right\rangle_{\mathcal{D}}+\left\langle\frac{H(A-M)}{M^{2}} \mid \delta \psi_{c}\right\rangle_{\mathcal{D}}=0
\end{gathered}
$$

Replacing $\mathcal{R}_{c}$ and $\mathcal{R}_{s}$ defined by (3) and (5) in Eq. (24), we obtain the following adjoint equations model:

$$
\begin{gathered}
{\left[\boldsymbol{\Omega} \cdot \boldsymbol{\nabla}+\mu_{t}(\boldsymbol{r})\right] \phi_{s}(\boldsymbol{r},-\boldsymbol{\Omega})=\mu_{s}(\boldsymbol{r}) \int_{\Omega^{\prime}=2 \pi} p\left(\boldsymbol{\Omega}^{\prime} \cdot(-\boldsymbol{\Omega})\right) \phi_{s}\left(\boldsymbol{r}, \boldsymbol{\Omega}^{\prime}\right) d \Omega^{\prime}-\mu_{a}(\boldsymbol{r}) \frac{(A(\boldsymbol{r})-M(\boldsymbol{r}))}{M^{2}},} \\
{\left[\boldsymbol{\Omega}_{c} \cdot \boldsymbol{\nabla}+\mu_{t}(\boldsymbol{r})\right] \phi_{c}(\boldsymbol{r})=\mu_{s}(\boldsymbol{r}) \int_{\Omega^{\prime}=2 \pi} p\left(\boldsymbol{\Omega}^{\prime} \cdot\left(-\boldsymbol{\Omega}_{\boldsymbol{c}}\right)\right) \phi_{s}\left(\boldsymbol{r}, \boldsymbol{\Omega}^{\prime}\right) d \Omega^{\prime}-\mu_{a}(\boldsymbol{r}) \frac{(A(\boldsymbol{r})-M(\boldsymbol{r}))}{M^{2}}}
\end{gathered}
$$

The directions $\boldsymbol{\Omega}$ and $\boldsymbol{\Omega}_{\boldsymbol{c}}$ were changed to $-\boldsymbol{\Omega}$ and $-\boldsymbol{\Omega}_{\boldsymbol{c}}$ for convenience.

If the tissue surfaces are assumed to be semi-transparent with specular reflection, the boundary conditions of the first equation are given by [39]:

$$
\phi_{s}(\boldsymbol{r}, \boldsymbol{\Omega})=\rho\left(\Theta_{s p}\right) \phi_{s}\left(\boldsymbol{r},-\boldsymbol{\Omega}_{\boldsymbol{s p}}\right) \text { for }(\boldsymbol{r}, \boldsymbol{\Omega}) \in \Gamma^{+} \text {with } \cos \Theta_{s p}=\boldsymbol{\Omega}_{\boldsymbol{s p}} \cdot \boldsymbol{n} \text { and } \cos \Theta=\boldsymbol{\Omega} \cdot \boldsymbol{n} \text {. }
$$


It can be seen that the adjoint equations model takes a similar form to the forward model. The adjoint equations model can be solved in a similar manner to that used to solve the forward model. It also shows that the first equation of (25) is solved to obtain $\phi_{s}(\boldsymbol{r}, \Omega)$ which is bring into the second equation to obtain $\phi_{c}(\boldsymbol{r})$.

\subsection{Gradient of the objective function}

The differentiation of the Lagrangian (for one fixed collimated source) with respect to $\theta$ in direction $\delta \theta$ satisfies:

$$
\frac{\partial \mathcal{L}\left(\theta, \psi_{c}, \psi_{s}, \phi_{c}, \phi_{s}\right)}{\partial \theta} \delta \theta=\frac{\partial J(\theta)}{\partial \theta} \delta \theta+\left\langle\phi_{c} \mid \frac{\partial \mathcal{R}_{c}}{\partial \theta} \delta \theta\right\rangle_{\mathcal{D}}+\left\langle\phi_{s} \mid \frac{\partial \mathcal{R}_{s}}{\partial \theta} \delta \theta\right\rangle_{\mathcal{D} \Omega}
$$

It should be noticed that the function $J$ (see Eqs. $(11,12)$ ) depends explicitly on $\theta$ only if $\theta=\mu_{a}$. For the other optical coefficients, $\frac{\partial J(\theta)}{\partial \theta}=0$. Using (10) and Eq. (20), Eq. (27) is reduced to:

$$
\langle\nabla J(\theta) \mid \delta \theta\rangle_{\mathcal{D}}=\left\langle\phi_{c} \mid \frac{\partial \mathcal{R}_{c}}{\partial \theta} \delta \theta\right\rangle_{\mathcal{D}}+\left\langle\phi_{s} \mid \frac{\partial \mathcal{R}_{s}}{\partial \theta} \delta \theta\right\rangle_{\mathcal{D} \Omega}\left(+\left\langle\frac{\Phi(A(\theta)-M)}{M^{2}} \mid \delta \theta\right\rangle_{\mathcal{D}} \text { if } \theta=\mu_{a}\right) .
$$

This is the expression that evaluates the gradient of the objective function. Applying respectively Eq. (28) to $\theta=\mu_{a}, \theta=\mu_{s}$ and $\theta=g$ we deduce the objective function gradient, with respect to these parameters:

$$
\begin{gathered}
\nabla J\left(\mu_{a}\right)=\frac{\Phi\left(A\left(\mu_{a}\right)-M\right)}{M^{2}}+\phi_{c} \psi_{c}+\left\langle\phi_{s} \mid \psi_{s}\right\rangle_{\Omega^{\prime}} \\
\nabla J\left(\mu_{s}\right)=\phi_{c} \psi_{c}+\left\langle\phi_{s} \mid \psi_{s}\right\rangle_{\Omega}-\left\langle\phi_{s} \mid \int_{\Omega^{\prime}=2 \pi} p\left(\boldsymbol{\Omega}^{\prime} \cdot \boldsymbol{\Omega}\right) \psi_{s}\left(\boldsymbol{r}, \boldsymbol{\Omega}^{\prime}\right) d \Omega^{\prime}+p\left(\boldsymbol{\Omega}_{\boldsymbol{c}} \cdot \boldsymbol{\Omega}\right) \psi_{c}(\boldsymbol{r})\right\rangle_{\Omega}, \\
\nabla J(g)=-\left\langle\phi_{s} \mid \mu_{s}(\boldsymbol{r})\left(\int_{\Omega^{\prime}=2 \pi} \frac{\partial p\left(\boldsymbol{\Omega}^{\prime} \cdot \boldsymbol{\Omega}\right)}{\partial g} \psi_{s}\left(\boldsymbol{r}, \boldsymbol{\Omega}^{\prime}\right) d \Omega^{\prime}+\frac{\partial p\left(\boldsymbol{\Omega}_{\boldsymbol{c}} \cdot \boldsymbol{\Omega}\right)}{\partial g} \psi_{c}(\boldsymbol{r})\right)\right\rangle_{\Omega} .
\end{gathered}
$$

It should be noticed that if more than one collimated sources are considered, the objective function gradient is obtained by summing the objective function gradient for each collimated source.

\subsection{Parameter and data scaling}

In order to speed-up the iterative convergence to the local minimum, a scaling strategy of the optical parameters [16] in the RTE based forward model was carried out in this work. Choosing an a priori function for each optical 
parameters, say $\mu_{a}^{r}, \mu_{s}^{r}, g_{r}$, the parameters are searched that fluctuate about unity. This scaling leads to recover:

$$
\sigma_{a}(\boldsymbol{r})=\frac{\mu_{a}(\boldsymbol{r})}{\mu_{a}^{r}(\boldsymbol{r})}, \sigma_{s}(\boldsymbol{r})=\frac{\mu_{s}(\boldsymbol{r})}{\mu_{s}^{r}(\boldsymbol{r})}, q(\boldsymbol{r})=\frac{g(\boldsymbol{r})}{g_{r}(\boldsymbol{r})},
$$

for which magnitude is of order one approximately for all these three new parameters. It results that the considered objective function becomes $J\left(\sigma_{a}, \sigma_{s}, q\right)$ (instead of $J\left(\mu_{a}, \mu_{s}, g\right)$ ). In the results presented further, the a priori functions for each optical parameters were chosen as those of the background (homogeneous) medium. The objective function gradients with respect to $\sigma_{a}, \sigma_{s}$ and $q$ are:

$$
\begin{gathered}
\nabla J\left(\sigma_{a}\right)=\mu_{a}^{r}(\boldsymbol{r}) \nabla J\left(\mu_{a}\right) ; \nabla J\left(\sigma_{s}\right)=\mu_{s}^{r}(\boldsymbol{r}) \nabla J\left(\mu_{s}\right) ; \\
\nabla J(q)=-\left\langle\phi_{s} \mid \mu_{s}(\boldsymbol{r})\left(\int_{\Omega^{\prime}=2 \pi} \frac{\partial p\left(\boldsymbol{\Omega}^{\prime} \cdot \boldsymbol{\Omega}\right)}{\partial q} \overline{\psi_{s}\left(\boldsymbol{r}, \boldsymbol{\Omega}^{\prime}\right)} d \Omega^{\prime}+\frac{\partial p\left(\boldsymbol{\Omega}_{\boldsymbol{c}} \cdot \boldsymbol{\Omega}\right)}{\partial q} \overline{\psi_{c}(\boldsymbol{r})}\right)\right\rangle_{A \Omega}
\end{gathered}
$$

A second scaling, as in [46], was necessary for the simultaneous reconstruction of three optical parameters distributions. Indeed, the optical coefficients to be reconstructed from the absorbed energy density are different in nature, and their order of magnitude also differs. As a consequence, the objective function gradient parts associated with these optical coefficients also differ by roughly the same order of magnitude, which is very bad for the convergence in the optimization problem when using a gradient-based method. Then, the objective function gradients with respect to $\sigma_{a}, \sigma_{s}$ and $q$ was scaled as:

$$
\nabla J^{\text {scaled }}\left(\sigma_{a}\right)=c_{\sigma_{a}} \nabla J\left(\sigma_{a}\right) ; \nabla J^{\text {scaled }}\left(\sigma_{s}\right)=c_{\sigma_{s}} \nabla J\left(\sigma_{s}\right) \text { and } \nabla J^{\text {scaled }}(q)=c_{q} \nabla J(q)
$$

where $c_{\sigma_{a}}, c_{\sigma_{s}}$ and $c_{q}$ are empirical coefficients that are determined after the first inverse iteration such that the largest element of the scaled gradient vector $\nabla J^{\text {scaled }}\left(\sigma_{a}\right)$ equals $5 \%$ of the largest element of vector $\sigma_{a}^{0}$ :

$$
c_{\sigma_{a}}=0.05 \frac{\max \left(\sigma_{a}^{0}\right)}{\max \left(\left|\nabla J\left(\sigma_{a}^{0}\right)\right|\right)}
$$


The same holds for $c_{\sigma_{s}}$. The results show that the best quality reconstruction are obtained when the largest element of $\nabla J^{\text {scaled }}(q)$ equals $1.5 \%$ of the largest element of $q^{0}$. The scaling factors are kept constant during the reconstruction from the first iteration.

In QPAT, where the dynamic range of the measured light intensities can be very large, scaling of the data may be needed in order to ensure numerical stability of the optimization problem. Furthermore, in this work, the data space was scaled similarly as in [16], where we used the logarithm of amplitude as the data.

\subsection{Implementation of the reconstruction algorithm}

A Modified Finite Volume Method (MFVM) of high accuracy [42] was used for solving the equations of the forward and adjoint models. This MFVM can be applied to arbitrarily shaped geometries, by using unstructured triangular grids. The methodology of the employed method is not repeated here, we refer the reader to [42] for comprehensive details. The objective function $J$ was iteratively minimized using the quasi-Newton algorithm with Lm-BFGS (limited-memory Broyden-Fletcher-Goldfarb-Shanno) [47]. It iteratively updates an initial estimate of the parameters distribution along a search descent direction denoted $d$. Once the minimum is found, the final result is the unknown parameters distribution. The updating procedure is formulated as: $\boldsymbol{\theta}^{k+1}=\boldsymbol{\theta}^{k}+\alpha^{k} d\left(\nabla J\left(\theta^{k}\right)\right)$ [47], where $k$ is the current iteration of the inverse procedure and $\alpha^{k}$ represents the step size obtained by the Armijo line search in order to provide a sufficient minimization of the objective function.

\section{Results and discussion}

We consider a 2D numerical phantom with a homogeneous background containing different inserts. In the first test case, the reconstruction were performed on a relatively large object of size $20 \times 20 \mathrm{~mm}^{2}$ while the other test cases use a phantom of $10 \times 10 \mathrm{~mm}^{2}$ (in all the simulations the length unit is the minimeter). The optical properties of the background are fixed to $\mu_{a}=0.05 \mathrm{~mm}^{-1}, \mu_{s}=5 \mathrm{~mm}^{-1}$ and $g=0.9$ expect for the final test case wherein $\mu_{s}$ and $g$ are assigned the value $6 \mathrm{~mm}^{-1}$ and 0.8 , respectively. The optical background values were used to start the optimization procedure by assuming a homogeneous medium. The geometry and positions of the inserts differ for each test in order to carry out different situations that highlight the main issues encountered in QPAT. The intensity 
of the Laser beam has a spatial Gaussian distribution along the $x$ - axis or $y$ - axis ( $s=x$ or $y$ ) such as:

$$
\Upsilon(s)=\frac{1}{\sigma_{s} \sqrt{2 \pi}} \exp \left(\frac{-\left(s-s_{c}\right)^{2}}{2 \sigma_{s}^{2}}\right),
$$

where $\sigma_{s}=1 \mathrm{~mm}$ is the standard deviation of the Laser beam. The position $s_{c}$ corresponds to the source location at the center of the illuminated wall of the medium. The angular space was discretized with 32 control solid angles whereas the number of nodes of the spatial mesh is given for each test case presented further. The quality of the algorithm is assessed thanks to the relative estimation error $\varepsilon$ between the retrieved $\hat{\beta}$ and the exact vector $\beta^{*}$ :

$$
\varepsilon=100 \% \frac{\left\|\hat{\boldsymbol{\beta}}-\boldsymbol{\beta}^{*}\right\|_{2}}{\left\|\boldsymbol{\beta}^{*}\right\|_{2}} \text { where }\|\cdot\|_{2} \text { denotes Euclidian norm. }
$$

\subsection{Anisotropy factor reconstruction}

This case points out the ability of the QPAT to reconstruct, in the multiple scattering regime, the anisotropy factor as an endogenous optical property of tissues. The spatial domain is discretized into 15,857 mesh nodes. Figure 1a illustrates the reference medium including three circular inserts centered at $(4 \mathrm{~mm} ;-2 \mathrm{~mm}),(10 \mathrm{~mm} ; 4 \mathrm{~mm})$ and $(16 \mathrm{~mm} ;-4 \mathrm{~mm})$ with a radius of $2 \mathrm{~mm}$. In this case, the absorption coefficient $\mu_{a}=0.05 \mathrm{~mm}^{-1}$ is kept constant while $\mu_{s}$ and $g$ are chosen so that their values lead to a constant value of $\mu_{s}^{\prime}=0.5 \mathrm{~mm}^{-1}$ in the whole phantom. This configuration avoids attributing the spatial variation of $g$ to $\mu_{s}^{\prime}=\mu_{s}(1-g)$. One Laser source was used to illuminate the west surface $(x=0 \mathrm{~mm})$. Figure $1 \mathrm{~b}$ shows the reconstructed image of the anisotropy factor. It can be seen that the retrieved image presents a good agreement with the reference object. The three inserts were spatially well fitted with their original positions. The estimated mean values inside the inclusions are correctly retrieved with respect to their exact values, even though the red-insert presents a slightly over-estimated values $\hat{g}_{\max }=0.96$ against $g^{*}=0.95$. The circular shape is also well reconstructed. This result implies that the spatial variation of $g$ cannot be caused by a variation of $\mu_{s}^{\prime}$ itself. The anisotropy factor can hence be independently reconstructed and separated from $\mu_{s}$ with our inverse algorithm. 

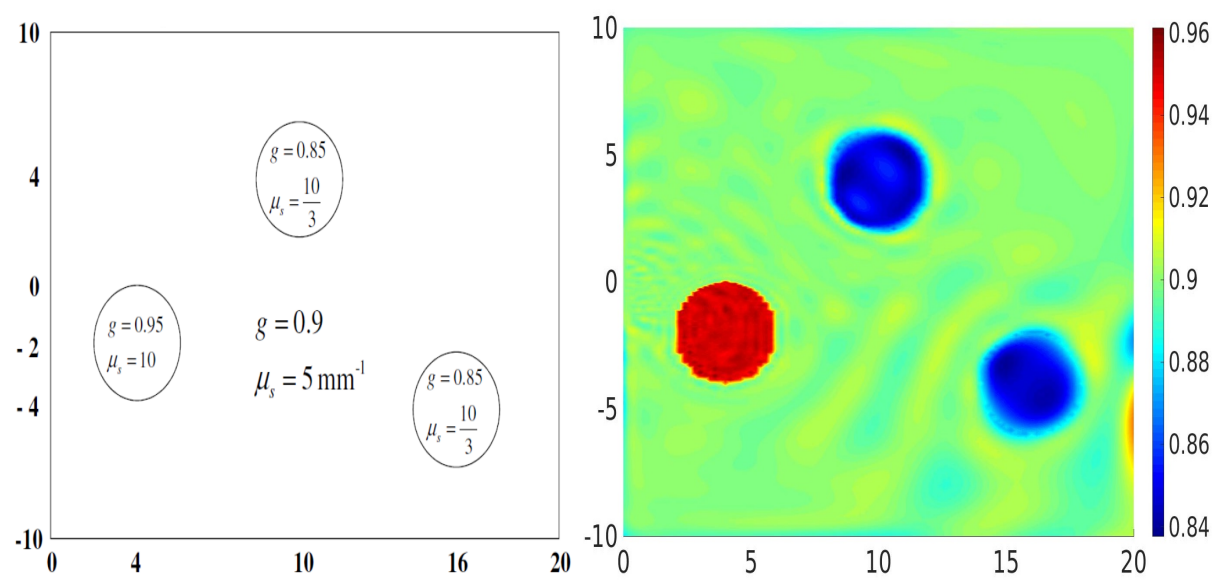

Figure 1: Reconstruction of the anisotropy factor: (a) the reference medium containing three inclusions at different spatial positions and (b) the reconstructed image of $g$.

\subsection{Source number effect}

After testing the capability of the proposed algorithm to reconstruct the anisotropy factor with one Laser source, we attempt in this case to assess the robustness of the QPAT to simultaneously reconstruct the three optical properties $\mu_{a}, \mu_{s}$ and $g$. This task has not been reported in the previous optical imaging related works, for our best knowledge. The original medium contains two circular inserts centered at $(2 \mathrm{~mm} ; 2 \mathrm{~mm})$ and $(-2 \mathrm{~mm} ;-2 \mathrm{~mm})$ with a radius of $1 \mathrm{~mm}$. The exact optical values of the top-right and bottom-left inclusions are assigned as $\mu_{a}=0.06 \mathrm{~mm}^{-1}, \mu_{s}$ $=6 \mathrm{~mm}^{-1}, g=0.85$ and $\mu_{a}=0.04 \mathrm{~mm}^{-1}, \mu_{s}=4 \mathrm{~mm}^{-1}$ and $g=0.95$, respectively. The unstructured triangular mesh used is composed of 2,821 nodes. Two illumination configurations were carried out: in the first one, the west surface was illuminated with one Laser source while in the second, the phantom is sequentially illuminated on its fourth tissue surfaces. The calculations were carried out with an Intel Xeon Processor E5-2683v4, 2.1GHz, 32 cores. This last uses Hyper-Threading and Intel C compiler. The computational time for the reconstruction in the second configuration was $40 \mathrm{~min}$. where 103 iterations were required. It can be noticed that, while keeping the same quality of reconstruction, the logarithmic scaling allowed to reduce by about a factor four the number of iterations and decreased the norms of the objective function gradients (with respect to each parameter to be recover) by a factor $10^{4}$. The obtained results for the first and second configurations are depicted in the left and right column of figure 2, respectively. Despite the critical inversion conditions concerning the large unknowns number of parameters $(3 \times 2,821)$ with only one source, the algorithm was still able to reveal the heterogeneities 
in the medium. In addition, the retrieved local values are close to their exact values even for the relatively deeper insert (top-right). The localization and the circular edge were achieved with a better quality reconstruction for the $\mu_{a}$ coefficient compared to those of the $\mu_{s}$ coefficient and anisotropy factor. This is due to the measured absorbed energy density used for fitting that is directly related to the $\mu_{a}$ coefficient which explains, therefore, the superior quality estimation for $\mu_{a}$ (see Eq.10). From the right column, the reconstructed images were significantly improved and the estimated values and the localization are in a good agreement with the real solution. The relative estimation errors for the top-right inclusion have been decreased from $8.9 \%, 10.45 \%$ and $3.65 \%$ to $0.17 \%, 2.37 \%$ and $0.75 \%$ for $\mu_{a}, \mu_{s}$ and $g$ respectively when illuminating the medium with four Laser sources. Furthermore, the circular shape is correctly reconstructed for all the optical parameters. This configuration has led to increasing the amount of measured data in the inverse procedure which allows thus to better reconstruct the optical properties simultaneously.

This result highlights the potential interest of using multiple sources which indeed corresponds to real experimental scenarios with a tomographic context. It is worth noting that the simultaneous reconstruction of the three optical parameters is not possible with the standard optical tomography since its inverse problem is usually under-determined and the measured data are collected on the tissue-surface. 

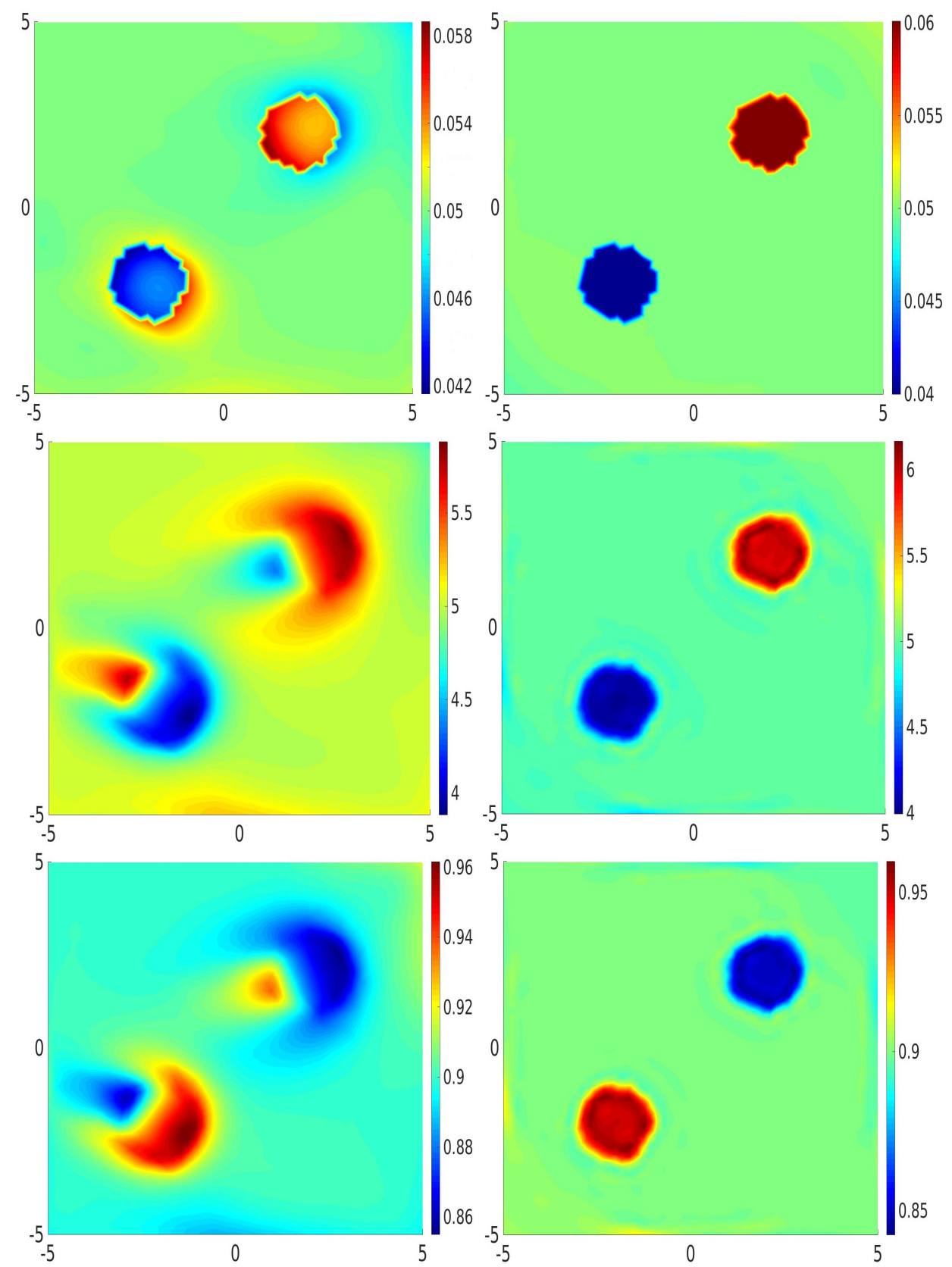

Figure 2: Simultaneous reconstruction of $\mu_{a}, \mu_{s}$ and $g$ with: (left column) only one Laser source illuminating the west surface and (right column), four Laser sources illuminating sequentially the phantom, (top raw) absorption coefficient $\mu_{a}$, (middle raw) scattering coefficient $\mu_{s}$ and (bottom raw) anisotropy factor $g$.

\subsection{Effect of anisotropy factor kept as a fixed constant}

We consider the second configuration as in the previous test case by assuming that the two inclusions represent heterogeneities only in $\mu_{a}$ and $\mu_{s}$ coefficients and the anisotropy factor $g$ is fixed at a constant value in the whole medium. The value of $g$ is most often in the range [0.8 - 1]. We then chose four different values of $g$ to represent this interval $(0.8,0.85,0.9$ and 0.95$)$. Figure 3 displays the reconstructed images of $\mu_{a}$ and $\mu_{s}$ for each fixed value of $g$. 
The relative estimation errors of the two inclusions for $\mu_{a}$ and $\mu_{s}$ are shown in Table 1. Qualitatively, the inclusions in $\mu_{a}$ and $\mu_{s}$ are recovered with low contrast level for $g=0.8$ and $g=0.85$ (due to the high backscattering of light) while the reconstruction is relatively improved when the $g$ values are increased to 0.9 and 0.95 . This has an important realistic interest because the biological tissues are known to be highly forward scattering. Therefore, more pronounced local artifacts are appeared near to the detectors and in the background especially in the $\mu_{s}$ images. For the four values of $g$, the $\mu_{a}$ coefficient is correctly retrieved with respect to the exact values of the two inclusions ( $\sim 0.06$ for the top-right and $\sim 0.04$ for the bottom-left). However, the reconstructed $\mu_{s}$ values become under- and over-estimated with respect to the inclusions-original values as the $g$ is higher since the two scattering parameters $\left(\mu_{s}\right.$ and $\left.g\right)$ are significantly correlated.

To assess the anisotropy factor effect, the obtained result in the previous test case (Fig. 2 right column) is compared with the present test case when $g$ is fixed at 0.9 in order to be closer as possible to the simulation conditions. For both cases, the background medium in the $\mu_{a}$ and $\mu_{s}$ images is recovered with the same quality (clear and homogeneous). The relative estimation errors for the inclusions of $\mu_{a}(0.11 \%$ for top-right and $0.13 \%$ for bottom-left $)$ is approximately similar while that of $\mu_{s}(46.47 \%$ for top-right and $45.64 \%$ for bottom-left) has been increased.

\begin{tabular}{ccccc}
\hline & \multicolumn{3}{l}{$g$ values } & \\
\cline { 2 - 5 }$\varepsilon(\%)$ & 0.8 & 0.85 & 0.9 & 0.95 \\
\hline$\mu_{a}$ (top-right inclusion) & 7.59 & 4.49 & 0.11 & 2.72 \\
$\mu_{a}$ (bottom-left inclusion) & 8.23 & 5.42 & 0.13 & 2.6 \\
$\mu_{s}$ (top-right inclusion) & 18.9 & 8.82 & 46.47 & 167.79 \\
$\mu_{s}$ (bottom-left inclusion) & 70.57 & 62.98 & 45.64 & 30.22 \\
\hline
\end{tabular}

Table 1: The relative estimation errors $\varepsilon$ of inclusions of $\mu_{a}$ and $\mu_{s}$ parameters for the four different values of $g$. 

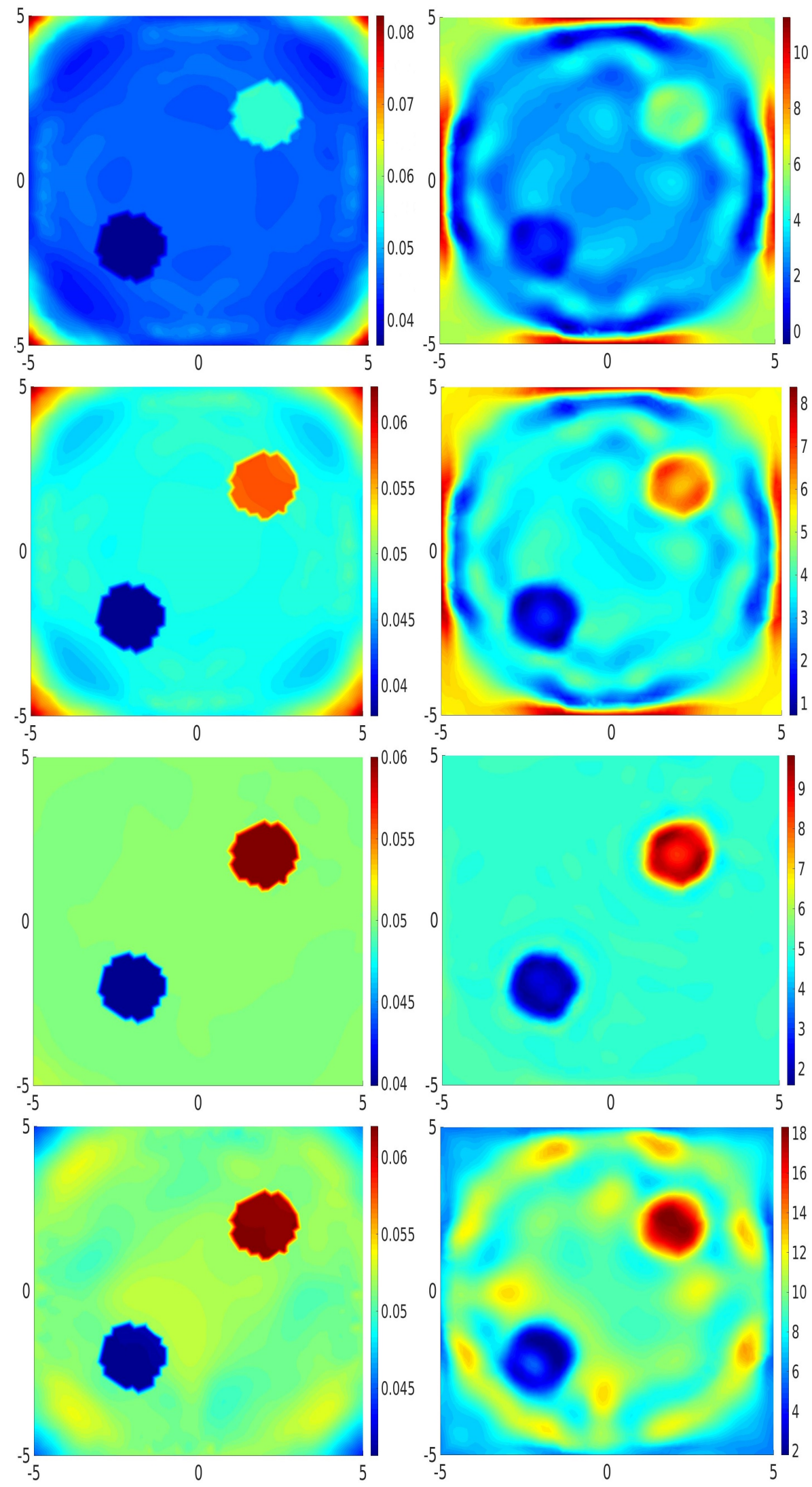

Figure 3: Simultaneous reconstruction of $\mu_{a}$ and $\mu_{s}$ when $g$ is kept as a fixed constant in the reconstruction procedure: (left column) absorption coefficient and (right column), scattering coefficient, (first line) $g=0.8$, (second line) $g=0.85$, (third line) $g=0.9$ and (last line) $g=0.95$. 


\subsection{Noise level effect}

In QPAT the measurements noise due to the experimental acquisition setup is unavoidable. To mimics real situations, the simultaneous reconstructions of $\mu_{a}, \mu_{s}$ and $g$ are performed using corrupted data at different noise levels of $1 \%, 3 \%$ and $6 \%$ added as a random Gaussian distribution on the exact predictions (absorbed energy density). The original phantom of section 4.2 illuminated by four Laser sources is used in this test case with the same spatial mesh. The used data (i.e. the absorbed energy density) with $6 \%$ of noise when the top wall is illuminated is depicted in Figure 4. The reconstructed images are shown in Figure 5.

The relative estimation errors of $\mu_{a}, \mu_{s}$ and $g$ parameters are given in table 2 for the three noise levels with also the noiseless case for comparison. They were computed over the whole reconstructed image domain. The obtained results show that our QPAT algorithm is able to localize the spatial positions of the inserts for the three parameters even with noisy data. As expected, it is seen that the image quality (characterized by its relative error) is worse as the noise level increases (see Tab. 2). Qualitatively, the artifacts and local perturbations become more pronounced and the circular shape of the inserts is degraded especially for $\mu_{s}$ and $g$ images. It can be seen that the $\mu_{a}$ images strongly handle the noise levels better than the scattering parameters $\mu_{s}$ and $g$. This is again explained by the fact that the fitted data in the inverse problem of the QPAT are directly dependent on the absorption coefficient. Quantitatively, the estimated mean values for the top-right insert become slightly over-estimated for $\mu_{a}$ and $\mu_{s}$ and under-estimated for $g$ with the noise level. Concerning the bottom-left insert, the retrieved mean values are slightly under-estimated for $\mu_{a}$ and $\mu_{s}$ and over-estimated for $g$ when the noise increases.

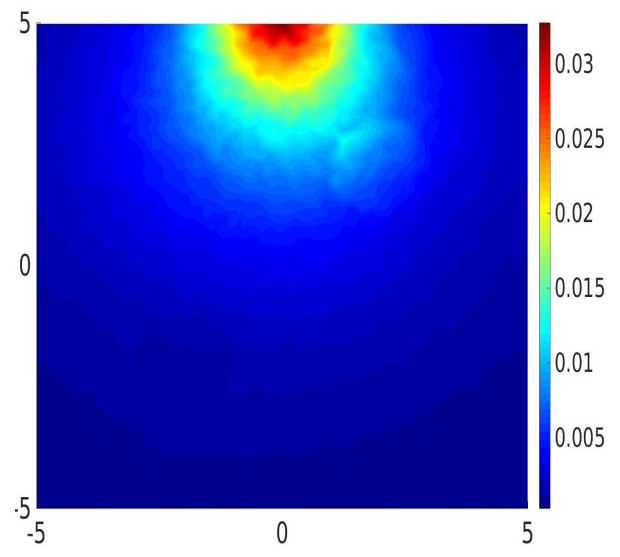

Figure 4: Used data (i.e. the absorbed energy density) with $6 \%$ of noise when the top wall is illuminated. 

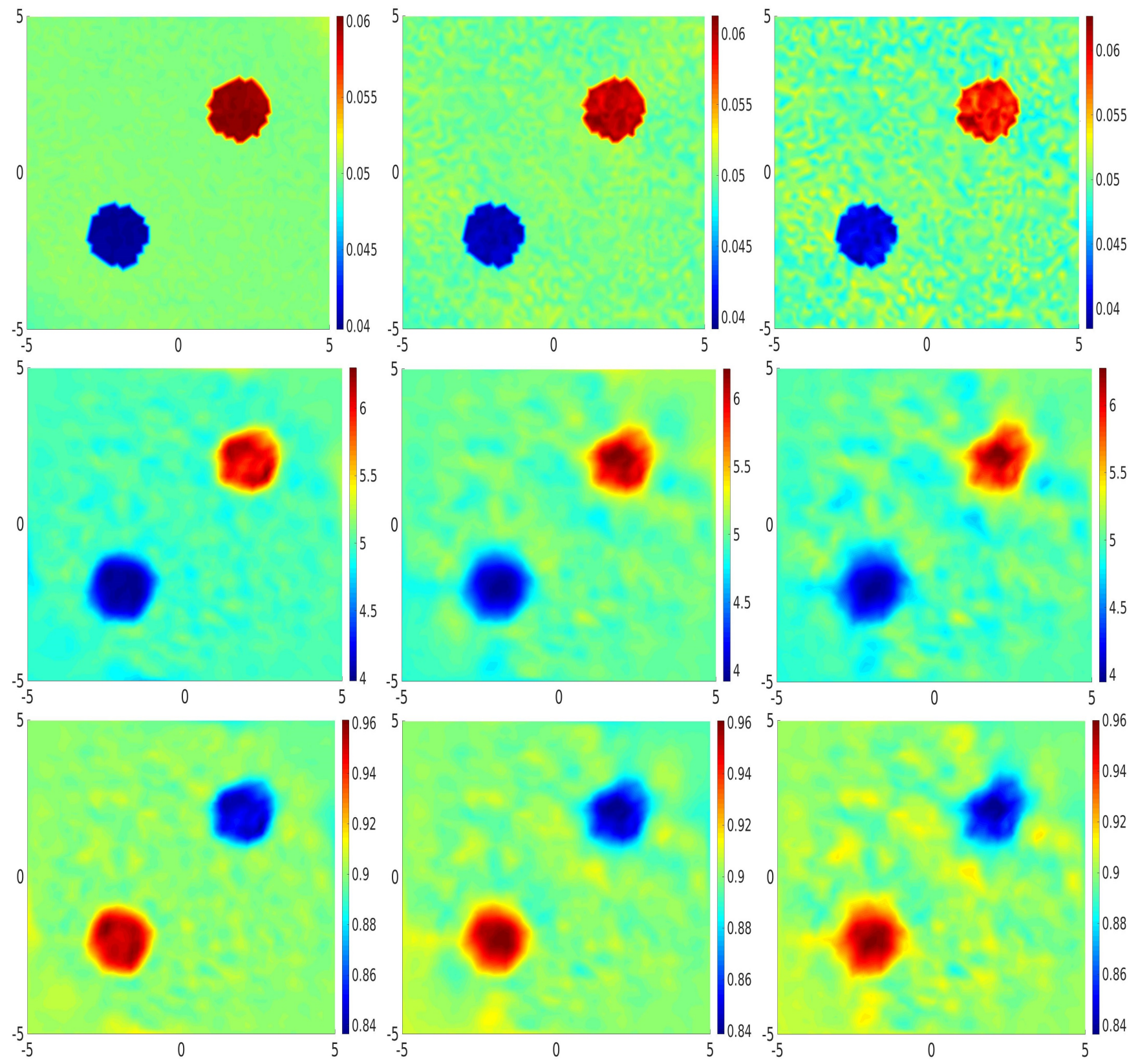

Figure 5: Simultaneous reconstruction with noisy data at different level of: (left column) $1 \%$, (middle column) $3 \%$ and (right column) $6 \%$ for the absorption coefficient $\mu_{a}$ (top raw), the scattering coefficient $\mu_{s}$ (middle raw) and the anisotropy factor $g$ (bottom raw).

\begin{tabular}{ccccc}
\hline & \multicolumn{3}{l}{ Noise levels } & \\
\cline { 2 - 5 }$\varepsilon(\%)$ & $0 \%$ & $1 \%$ & $3 \%$ & $6 \%$ \\
\hline$\mu_{a}$ & 0.54 & 1.53 & 3.83 & 8.26 \\
$\mu_{s}$ & 7.63 & 10.50 & 13.03 & 14.24 \\
$g$ & 1.79 & 2.63 & 3.33 & 3.60 \\
\hline
\end{tabular}

Table 2: The relative estimation errors $\varepsilon$ of $\mu_{a}, \mu_{s}$ and $g$ parameters of the reconstruction algorithm for the four different noise levels on the absorbed density energy data. 


\subsection{Crosstalk effect}

The crosstalk problem is often encountered in optical imaging when reconstructing simultaneously the $\mu_{a}$ and $\mu_{s}$ coefficients. It is worth noting that the simultaneous reconstruction of $\mu_{a}, \mu_{s}$ and $g$ parameters has not been previously reported in the literature, for authors' best knowledge. In this case the crosstalk problem and the interparameter effects become challenger in the recovered images. In this work, we highlight the interest of the QPAT to simultaneously reconstruct the three optical properties while the conventional optical imaging fails to perform this task. We present a test case that mimics a crosstalk problem between $\mu_{a}, \mu_{s}$ and $g$ wherein their original images are depicted in Figs. 6a-c, respectively. The same spatial mesh that previously presented (2, 821 nodes) was used. The phantom was illuminated by four Laser sources. The corresponding recovered images are shown in Figs. 6d-e. The reconstructed results show that the crosstalk effect is only presented in the $\mu_{s}$ and $g$ images. The impact of the $g$-insert appears with a high contrast heterogeneity in the $\mu_{s}$ image $\left(\varepsilon_{\mu_{s}}^{\text {crosstalk }}=12 \%\right)$ while the $\mu_{s}$ insert produces, in turn, a small contrast heterogeneity in the $g$ image $\left(\varepsilon_{g}^{\text {crosstalk }}=1.56 \%\right)$. Therefore, the crosstalk error induced in the $\mu_{s}$ coefficient is more pronounced than that obtained for the anisotropy factor. This is due to the high sensitivity of this factor on the light scattering. On the other hand, none crosstalk effect was found in the $\mu_{a}$ image which is reconstructed in a very good agreement with its original image. Moreover, the $\mu_{a}$ inclusion has no impact on the $\mu_{s}$ and $g$ images. That can be explained by the fact the absorbed energy density is directly dependent on the $\mu_{a}$ coefficient, which makes the fitted data more sensitive for $\mu_{a}$ than the $\mu_{s}$ and $g$ parameters. Within the minimization scheme, when the algorithm is seeking to simultaneously reconstruct the three parameters $\left(\mu_{a}, \mu_{s}\right.$ and $g$ ), the $\mu_{a}$ image is accurately and quickly obtained after only few iterations of the convergence. Therefore, the $\mu_{s}$ and $g$ coefficients are reconstructed as in the case that assuming the $\mu_{a}$ coefficient is fixed to its exact value. The superior quality reconstruction of $\mu_{a}$, under these crucial situations, implies that the QPAT has an important interest for pre-clinical applications because the $\mu_{a}$ coefficient can lead to further physiological properties such as oxygen saturation, hemoglobin concentration, blood oxygenation, etc.

The crosstalk is a challenging problem in QPAT which has been reported in previous works related to optical imaging. The crosstalk is induced when the $\mu_{a}$ coefficient is only changed for a particular tissue due to physiological 
variation while its $\mu_{s}$ coefficient remains unchanged. Its practical implications can mainly concern the blood tumors or other tissues which not containing fibrous. The crosstalk assessment for cancer diagnosis can allow highlighting the robustness of the imaging system to handle the false positive tumoral inclusions. In order to overcome its effect, more data are needed in the reconstruction algorithm to reduce the uniqueness character of the inverse problem.
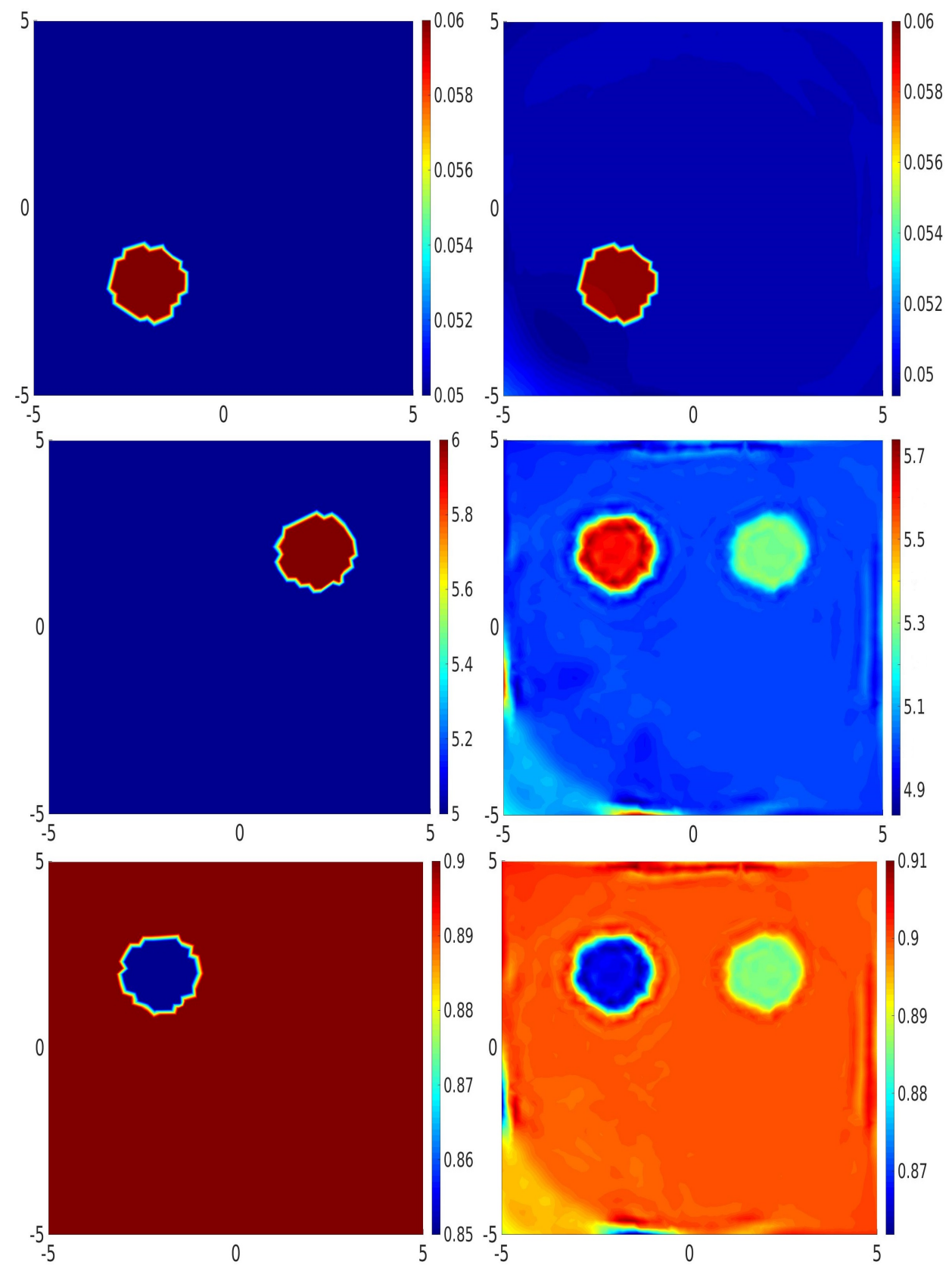

Figure 6: Simultaneous reconstruction of $\mu_{a}, \mu_{s}$ and $g$ with a crosstalk problem: (left column) original images and (right column) reconstructed images, (top raw) absorption coefficient $\mu_{a}$, (middle raw) scattering coefficient $\mu_{s}$ and (bottom raw) anisotropy factor $g$. 


\subsection{Slabs inserts reconstruction}

In this case, we assess the performance of the QPAT to reconstruct rectangular heterogeneities with different thicknesses and distance separations. To this end, two examples are studied. In the first example, four thin slabs of 200 $\mu \mathrm{m}$ of thickness separated by $2 \mathrm{~mm}$ are inserted in the phantom (see Fig. 7 top-raw). In the second example, three slabs have $1 \mathrm{~mm}$ of thickness with $50 \mu \mathrm{m}$ of separation (see Fig. 7 bottom-raw). The spatial mesh has been increased to 33,025 nodes for both cases to suitably represent the thin inserts and the small separation. The phantoms were illuminated by four Laser sources. The reconstructed results of the first and second example are shown in the left and right column of Fig. 8, respectively. Also, the reconstructions were achieved without a crosstalk problem.

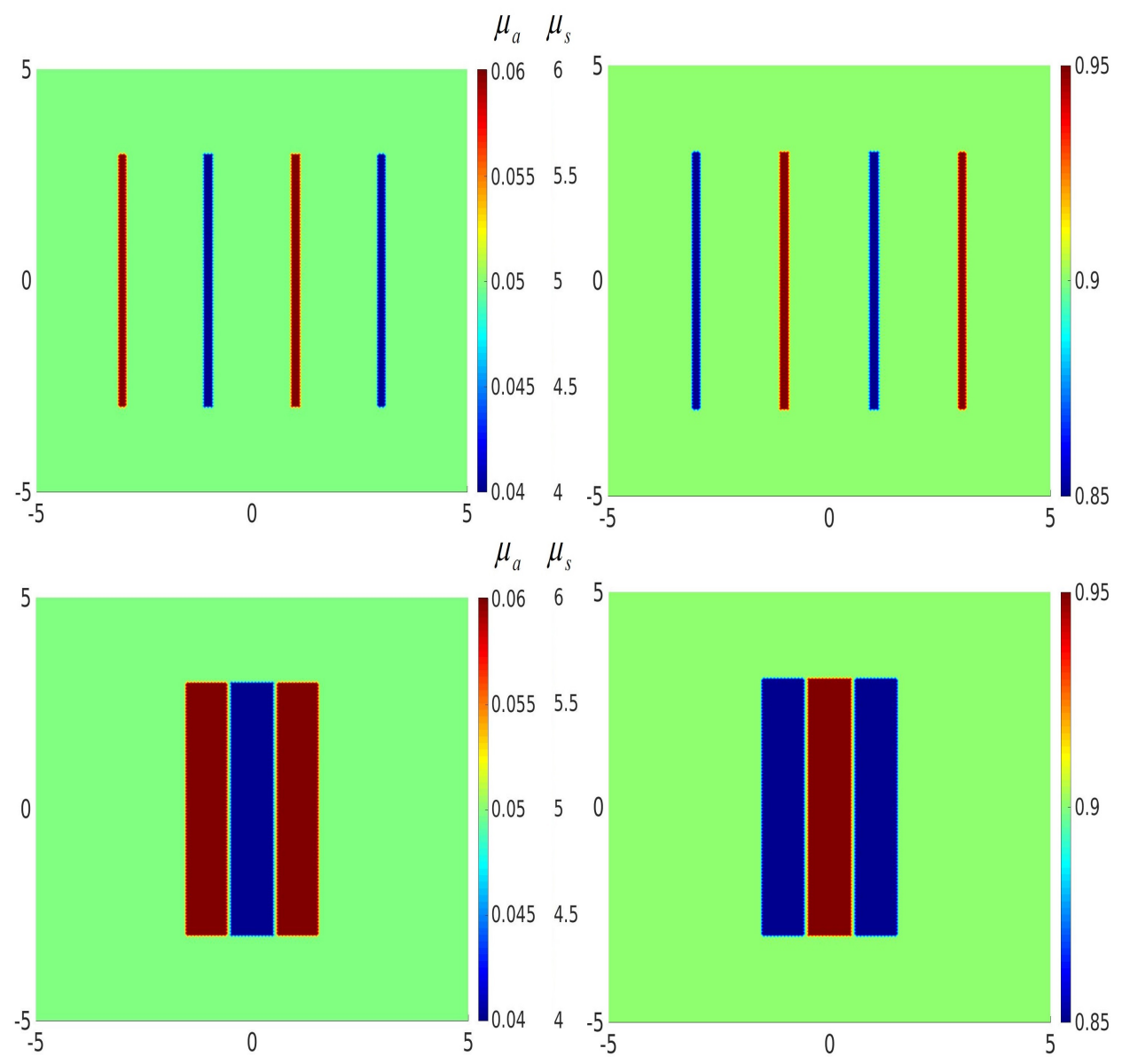

Figure 7: The original images containing the rectangular slabs inserts of the first example (top raw) and the second example (bottom raw). The right column shows the exact values of the anisotropy factor while the left column indicates the exact values for the $\mu_{a}$ and $\mu_{s}$ coefficients.

Figure 8 shows the robustness of our QPAT algorithm to accurately reconstruct the thin slabs heterogeneities (left column in Fig. 8) and also to precisely separate the small-inter-distance thick inserts (right column in Fig. 8). The 
algorithm is able to retrieve the localization, the size, the thicknesses and also the local optical values $\mu_{a}, \mu_{s}$ and $g$ of the rectangular slabs inserts. Therefore, the reconstruct images were achieved with a good quantitative and qualitative accuracy. For both examples, these slabs are recovered with a high contrast level, contrary to optical imaging. The use of the local absorbed energy density, related to the initial acoustic pressure, has advantageously provided a potential improvement of the spatial resolution to the optical properties images.
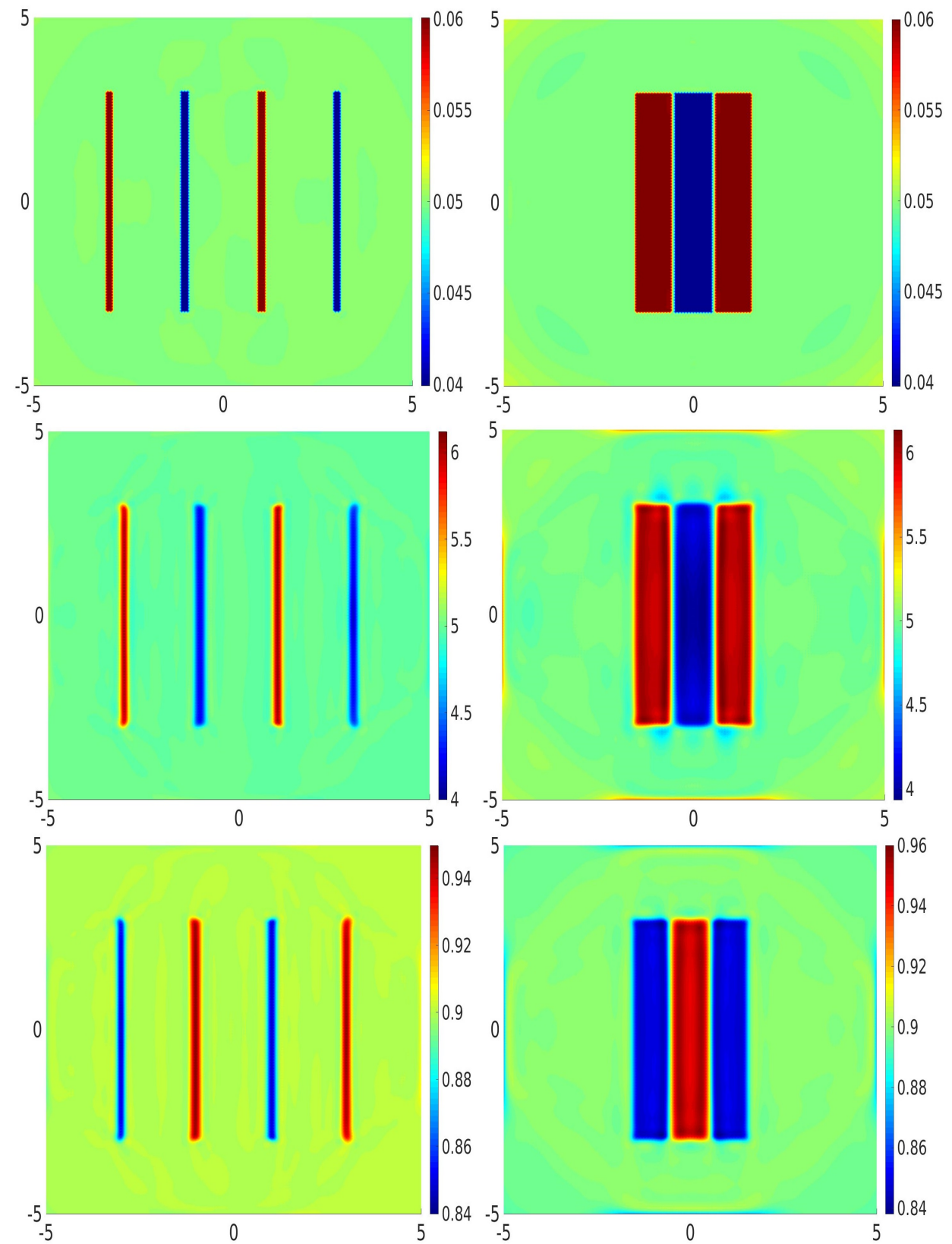

Figure 8: The reconstructed images of: (left column) the first example and (right column) the second example, (top raw) the absorption coefficient $\mu_{a}$, (middle raw) the scattering coefficient $\mu_{s}$ and (bottom raw) the anisotropy factor $g$. 


\section{Conclusion}

The optical inverse problem of the QPAT using the RTE as light transport model was presented. The adjoint method was applied to efficiently compute the gradient of the objective function where its expressions for $\mu_{a}, \mu_{s}$, and $g$ coefficients were explicitly obtained. For the first time, the simultaneous reconstruction of $\mu_{a}, \mu_{s}$, and $g$ was possible thanks to our QPAT algorithm that uses spatially absorbed energy density data. The results showed that treating the anisotropy parameter as a fixed constant leads to low errors in the reconstructed images of the absorbing coefficient and significant errors in the reconstructed images of the scattering coefficient. Also, they showed that the $\mu_{a}$ images are reconstructed with a better estimation quality than $\mu_{s}$ and $g$ images even with noisy data or when only one Laser source was used. Furthermore, the $\mu_{a}$ images are insensitive to the crosstalk issue contrary to those of $\mu_{s}$ and $g$ parameters. The obtained results highlight the interest of the QPAT algorithm to advantageously complete the conventional imaging modalities for cancer diagnosis. This work was a necessary preliminary study to show that a complete optical imaging of tissue is possible through the QPAT modality. The extension of our method to 3D geometries for real applications is a significant numerical challenge which is not straightforward. We plan to investigate this problem.

\section{Appendix A. Calculations of adjoint operators}

It should be noted that in the calculations given below, the quantities are not divided by $M^{2}$ as in (22) for the sake of simplicity. This does not change the result. From (12), we have:

$$
\begin{aligned}
& \left\langle A-M \mid\left(\widetilde{H} \delta \psi_{s}\right)\right\rangle_{\mathcal{D}}=\int_{\mathcal{D}}(A-M)(\boldsymbol{r}) \mu_{a}(\boldsymbol{r}) \int_{\Omega=2 \pi} \delta \psi_{s}(\boldsymbol{r}, \boldsymbol{\Omega}) d \Omega d r \\
= & \int_{\mathcal{D}} \int_{\Omega=2 \pi}(A-M)(\boldsymbol{r}) \mu_{a}(\boldsymbol{r}) \delta \psi_{s}(\boldsymbol{r}, \boldsymbol{\Omega}) d \Omega d r=\left\langle A-M \mid\left(H \delta \psi_{s}\right)\right\rangle_{\mathcal{D} \Omega} \\
= & \int_{\mathcal{D}} \int_{\Omega=2 \pi} \mu_{a}(\boldsymbol{r})(A-M)(\boldsymbol{r}) \delta \psi_{s}(\boldsymbol{r}, \boldsymbol{\Omega}) d \Omega d r=\left\langle H^{*}(A-M) \mid \delta \psi_{s}\right\rangle_{\mathcal{D} \Omega} .
\end{aligned}
$$


Also,

$$
\begin{aligned}
& \left\langle A-M \mid\left(H \delta \psi_{c}\right)\right\rangle_{\mathcal{D}}=\int_{\mathcal{D}}(A-M)(\boldsymbol{r}) \mu_{a}(\boldsymbol{r}) \delta \psi_{c}(\boldsymbol{r}, \boldsymbol{\Omega}) d r \\
& \int_{\mathcal{D}} \mu_{a}(\boldsymbol{r})(A-M)(\boldsymbol{r}) \delta \psi_{c}(\boldsymbol{r}, \boldsymbol{\Omega}) d r=\left\langle H^{*}(A-M) \mid \delta \psi_{c}\right\rangle_{\mathcal{D}}
\end{aligned}
$$

389 Thus, $H^{*}=H$. 


\section{Bibliography}

[1] Pinkert M A, Salkowski L R, Keely P J, Hall T J, Block W F and Eliceiri K W 2018 Review of quantitative multiscale imaging of breast cancer J. Med. Imaging 5(1) 010901

[2] Anastasio M A, Beard P C and Zhu Q 2017 Photoacoustic imaging and sensing J. Biomed. Opt. 22(4) 041001

[3] Wang L V and Yao J 2016 A practical guide to photoacoustic tomography in the life sciences Nat. Meth. 13 $627-638$

[4] Xia J and Wang L V 2014 Small-animal whole-body photoacoustic tomography: a review Phys. Med. Biol. 61(5) 1380-1389

[5] Anastasio M A and Beard P C 2012 Photoacoustic imaging and sensing J. Biomed. Opt. 17(6) 061201

[6] Wang L V and Hu S 2012 Photoacoustic tomography: in vivo imaging from organelles to organs Science 335(6075) 1458-1462

[7] Cox B, Laufer J G, Arridge S R and Beard P C 2012 Quantitative spectroscopic photoacoustic imaging: a review J. Biomed. Opt. 17(6) 061202

[8] Beard P 2011 Biomedical photoacoustic imaging Interface Focus 1(4) 602-631

[9] Ntziachristos V 2010 Going deeper than microscopy: the optical imaging frontier in biology Nat. Meth. 7(8) 603

[10] Li C and Wang L V 2009 Photoacoustic tomography and sensing in biomedicine Phys. Med. Biol. 54(19) R59 
[11] Wang L V 2009 Photoacoustic imaging and spectroscopy, CRC Press, Florida

[12] Xu M and Wang L V 2006 Photoacoustic imaging in biomedicine Rev. Scient. Instum. 77(4) 041101

[13] Ntziachristos V, Ripoll J, Wang L V and Weissleder R 2005 Looking and listening to light: the evolution of whole-body photonic imaging Nat. Biotechnol. 23(3) 313

[14] Ripoll J 2012 Principles of diffuse light propagation. Light propagation in tissues with applications in biology and medicine (Singapore: World Scientific)

[15] Yao L, Sun Y and Jiang H 2010 Transport-based quantitative photoacoustic tomography: Simulations and experiments Phys. Med. Biol. 55(7) 1917-1934

[16] Tarvainen T, Cox B T, Kaipio J P and Arridge S R 2012 Reconstructing absorption and scattering distributions in quantitative photoacoustic tomography Inverse Probl. 28(8) 084009

[17] Saratoon T, Tarvainen T, Cox B T and Arridge S R 2013 A gradient-based method for quantitative photoacoustic tomography using the radiative transfer equation Inverse Probl. 29(7) 075006

[18] Jacques S L 2013 Optical properties of biological tissues: a review Phys. Med. Biol. 58(11) R37

[19] Wang Z, Tangella K, Balla A, Popescu G 2011 Tissue refractive index as marker of disease, J Biomed. Opt. 16(11) 116017

[20] Hillegersberg R V, Pickering J W, Maurice A, and Beek J F 1993 Optical properties of rat liver and tumor at $633 \mathrm{~nm}$ and 1064 nm: Photofrin enhances scattering Lasers Surg. Med. 13(1) 31-39

[21] Germer G T, Andre R, Ritz J P, Christoph I, Dirk A, Gerhard M and Buhr H J 1998 Optical properties of native and coagulated human liver tissue and liver metastases in the near infrared range Lasers Surg. Med. 23(4) 194-203

[22] Yao L, Sun Y and Jiang H 2009 Quantitative photoacoustic tomography based on the radiative transfer equation Opt. Lett. 34(12) 1765-1767 
[23] Bal G, Jollivet A and Jugnon V 2010 Inverse transport theory of photoacoustics Inverse Probl. 26025011

[24] Xi L, Li X, Yao L, Grobmyer S and Jiang H 2012 Design and evaluation of a hybrid photoacoustic tomography and diffuse optical tomography system for breast cancer detection Med. Phys. 39(5) 2584-2594

[25] Gao H, Osher S and Zhao H 2012 Quantitative photoacoustic Tomography Lecture Notes Math. 2035 131-158

[26] Mamonov A V and Ren K 2013 Quantitative photoacoustic imaging in the radiative transport regime Commun. Math. Sci. 12(2) 201-234

[27] Haltmeier M, Neumann L and Rabanser S 2015 Single-stage reconstruction algorithm for quantitative photoacoustic tomography Inverse Probl. 31(6) 065005

[28] De Cezaro A, Travessini De Cezaro F and Sejje Suarez J 2015 Regularization approaches for quantitative Photoacoustic tomography using the radiative transfer equation J. Math. Anal. Appl. 429(1) 415-438

[29] Pulkkinen A, Cox B T, Arridge S R, Kaipio J P and Tarvainen T 2015 Quantitative photoacoustic tomography using illuminations from a single direction J. Biomed. Opt. 20(3) 036015

[30] Li X, Heldermon C D, Yao L, Xi L and Jiang H 2015 High resolution functional photoacoustic tomography of breast cancer Med. Phys. 42(9) 5321-5328

[31] Hochuli R, Powell S, Arridge S R and Cox B 2016 Quantitative photoacoustic tomography using forward and adjoint Monte Carlo models of radiance J. Biomed. Opt. 21(12) 126004

[32] Tarvainen T, Pulkkinen A, Cox B T and Arridge S R 2017 Utilizing the radiative transfer equation in quantitative photoacoustic tomography Proc. of SPIE Vol. 10064, Photons Plus Ultrasound: Imaging and Sensing, 100643E-1

[33] Wang C and Zhou T 2017 On iterative algorithms for quantitative photoacoustic tomography in the radiative transport regime Inverse Probl. 33(11) 115006 
[34] Okawa S, Hirasawa T, Kushibiki T and Ishihara M 2017 Effects of the approximations of light propagation on quantitative photoacoustic tomography using two-dimensional photon diffusion equation and linearization Opt. Rev. 24(6) 705-726

[35] Haltmeier M, Neumann L, Nguyen L and Rabanser S 2018 Analysis of the linearized problem of quantitative photoacoustic tomography SIAM J. Appl. Math. 78(1) 457-478

[36] Bal G and Ren K 2011 Multi-source quantitative photoacoustic tomography in a diffusive regime Inverse Probl. 27075003

[37] Shao P, Cox B, and Zemp R J 2011 Estimating optical absorption, scattering, and Grueneisen distributions with multiple-illumination photoacoustic tomography Appl. Opt. 50(19) 3145-3154

[38] Addoum A, Contassot-Vivier S and Asllanaj F 2019 Three-dimensional frequency-domain optical anisotropy imaging of biological tissues with near-infrared light Med. Phys. 46(9) 4057-4069

[39] Asllanaj F, Addoum A and Roche J R 2018 Fluorescence molecular imaging based on the adjoint radiative transport equation Inverse Probl. 34075009

[40] Addoum A, Farges O and Asllanaj F 2018 Optical properties reconstruction using the adjoint method based on the radiative transfer equation J. Quant. Spectrosc. Radiat. Transfer 204 179-189

[41] Saratoon T, Tarvainen T, Arridge S R and Cox B T 2013 3D quantitative photoacoustic tomography using the delta-Eddington approximation Proc. of SPIE Vol. 8581, Photons Plus Ultrasound: Imaging and Sensing, $85810 \mathrm{~V}$

[42] Asllanaj F, Contassot-Vivier S, Liemert A and Kienle A 2014 Radiative transfer equation for predicting light propagation in biological media: comparison of a modified finite volume method, the Monte Carlo technique and an exact analytical solution J. Biomed. Opt. 19015002

[43] Klose A D 2009 Light Scattering. Reviews 4, Book Chapter: Radiative transfer of luminescence light in biological tissue (Berlin Heidelberg: Springer:293-345) 
[44] Fiacco A V and McCormick G P 1968 Nonlinear Programming: Sequential Unconstrained Minimization Techniques (John Wiley and Sons)

[45] Bonnans J F, Gilbert J C, Lemarechal C and Sagastizabal C A 2016 Numerical Optimization Theoretical and Practical Aspects (Springer Sciences and Business Media)

[46] Klose A 2001 Optical tomography based on the radiative transfer equation. PhD thesis, Department of Physics Freie Universitat, Berlin Germany

[47] Klose A D, Ntziachristos V and Hielscher A H 2005 The inverse source problem based on the equation of radiative transfer in optical molecular imaging J. Comp. Phys. 202 323-345 